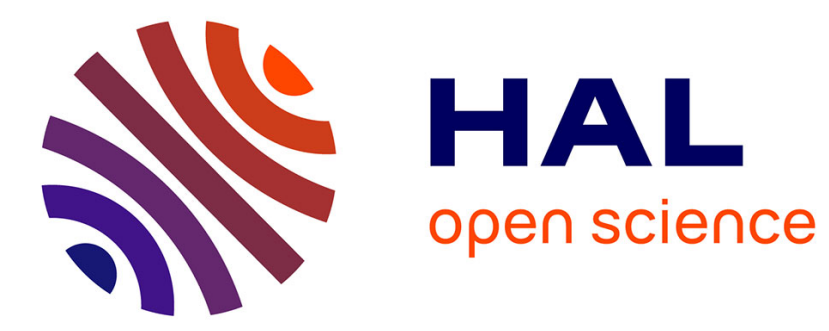

\title{
V2I-Based Architecture for Information Exchange among Vehicles
}

Vicente Milanés, Jorge Godoy, Joshué Pérez Rastelli, Blas Vinagre, Carlos González, Enrique Onieva, Javier Alonso

\section{- To cite this version:}

Vicente Milanés, Jorge Godoy, Joshué Pérez Rastelli, Blas Vinagre, Carlos González, et al.. V2IBased Architecture for Information Exchange among Vehicles. 7th IFAC Symposium on Intelligent Autonomous Vehicles, Sep 2010, Lecce, Italy. hal-00738034

\section{HAL Id: hal-00738034 https://hal.inria.fr/hal-00738034}

Submitted on 3 Oct 2012

HAL is a multi-disciplinary open access archive for the deposit and dissemination of scientific research documents, whether they are published or not. The documents may come from teaching and research institutions in France or abroad, or from public or private research centers.
L'archive ouverte pluridisciplinaire HAL, est destinée au dépôt et à la diffusion de documents scientifiques de niveau recherche, publiés ou non, émanant des établissements d'enseignement et de recherche français ou étrangers, des laboratoires publics ou privés. 


\title{
V2I-Based Architecture for Information Exchange among Vehicles
}

\author{
V. Milanés* J. Godoy* J. Pérez* B. Vinagre** C. González* \\ E. Onieva* J. Alonso* \\ * Centro de Automática y Robótica, Consejo Superior de \\ Investigaciones Científicas 28500 Madrid, Spain (e-mail: \\ vmilanes@iai.csic.es,jlgodoy@iai.csic.es,jperez@iai.csic.es, \\ gonzalez@iai.csic.es,onieva@iai.csic.es,jalonso@iai.csic.es). \\ ** Escuela de Ingenieros Industriales Universidad de Extremadura \\ 06006 Badajoz, Spain (e-mail: bvinagre@unex.es)
}

\begin{abstract}
The significant increment in the number of advanced driver assistance systems (ADAS) in mass-produced cars suggest the idea of autonomous or semi-autonomous vehicles driving in roads in a medium-large term. Consequently, a system to allow the communication among this kind of vehicles and manual driven vehicles will have to be used in order to permit the circulation of both of them at the same time. Two communication modes have to be taken into account. First, a vehicle-to-vehicle communication (V2V) to permit the traffic data flow among cars. Second, a vehicle-to-infrastructure communication (V2I) to allow a central station to coordinate the movements of the vehicle in case of a failure in the V2V. In this paper we present an architecture capable of operating either in autonomous vehicles or manual driven vehicles and coordinating their movements based on a V2I communication. Two mass-produced vehicles are used in order to test the behavior of the architecture implementation. Experimental trials to study the response of the system and the information flow have been carried out.
\end{abstract}

Keywords: Autonomous vehicles, Control stations, Communication systems, Information integration, Local area networks.

\section{INTRODUCTION}

In the present, the technological advances incorporated to the automobile sector have caused a change in the driving style of the drivers. Systems as the adaptive cruise control (ACC) (Vahidi and Eskandarian, 2003), based on radar technology, that enables the driver to preselect a suitable road speed or as the lane keeping assist (LKA) (Wu et al., 2008), based on vision system that helps drivers to maintain their vehicle within the lane, permit reducing the influence of the human factor increasing the dependence on ADAS systems. So, drivers are being saved of the more tedious driving task so they can be performed by the automatic systems.

These advances, in our opinion, will cause the coexistence of vehicles that can be guided through automatic controllers and vehicles driven manually in a large-medium term. Furthermore, a system that permits the communication among all the vehicles with independence of the way they are driven is needed. V2V communication is a good solution to send data coming from different sensors among vehicles. However, manual vehicles are not equipped with this ability and V2I communication is needed to send

\footnotetext{
* This work has been developed thanks to the Spain's Ministerio de Fomento through the GUIADE (P9/08) project and the Plan Nacional from the Spanish Goverment with the TRANSITO (TRA200806602-C03-01) project.
}

information about its position, direction and intention without modifying the indoor of the manual vehicles.

$\mathrm{V} 2 \mathrm{~V}$ has been widely studied and different solutions has been proposed (Chen and Cai, 2005; Biswas et al., 2006) because of its decisive contribution in the increment of the safety. Nowadays, the development of Dedicated Short Range Communications (DSRC) as a reserved band for the communications among vehicles, suggest the importance of applying communications system to increase the safety in the roads.

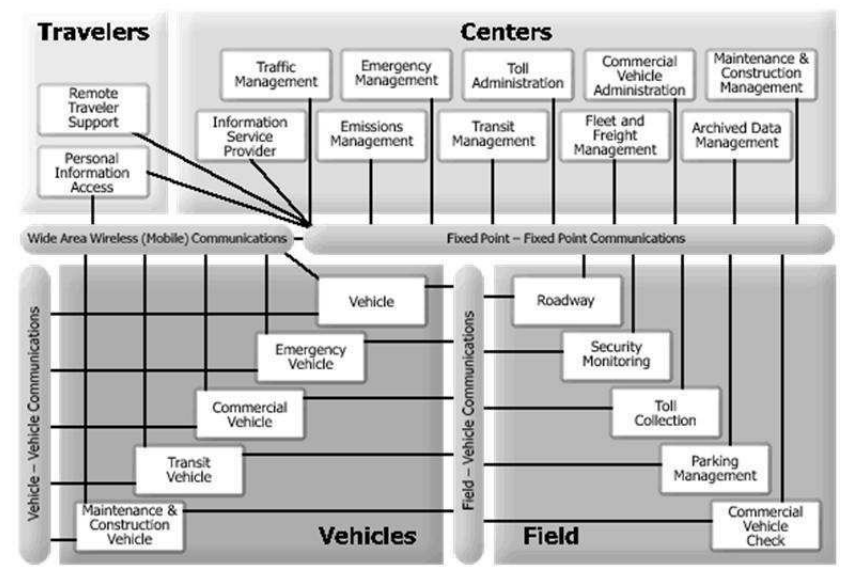

Fig. 1. ITS architecture of the Department of Transportation (USA). 
Within vehicle safety communications (VSC), significant advances are being underway around the world. The Department of Transportation in the United States defined in 2006 the IEEE 1609 Family of Standards for Wireless Access in Vehicular Environments (WAVE). This standard is composed by three main elements: the On Board Unit (OBU), Road Side Unit (RSU) and WAVE interface. Based on this principle, a communications model, management structure, security mechanisms and physical access for wireless communications in the vehicular environment were developed. In a parallel line of work, this department proposed an architecture (Fig. 1) for the development of Intelligent Transportation Systems. Four main components are defined (travelers, centers, vehicles and field) that are interconnected through four different types of communications.

In the European Union, the Intelligent Car Initiative includes the e-safety initiative. The main goal is to achieve Intelligent Vehicle Safety Systems (IVSS) that use information and communication technologies to increase road safety and reduce the number of accidents on Europe's roads. Co-operative Systems for Intelligent Road Safety (COOPERS) project (Piao and McDonald, 2008) is included in e-safety. COOPERS's aim is to improve the safety of the road directing and updating the communication of the information of traffic between the infrastructure and the vehicles in a section of the road. To this end, it evaluates the different communications technologies capable of applying in a $\mathrm{I} 2 \mathrm{~V}$ bidirectional communication. Also included in the EU 6th Framework Program (FP), the Co-operative Vehicle-Infrastructure Systems (CVIS) project (Singh et al., 2004) tried to specify an architecture of communication and a set of applications for communications Vehicle-Road and Vehicle-Internet. This architecture is based on the architecture CALM, ISO standard. Another project focused on the same line is the Cooperative systems for road safety "Smart Vehicles on Smart Roads" (SAFESPOT) (Vivo et al., 2007). It is a project to study how vehicles and intelligent roads can cooperate to increase the safety. The aim is to anticipate accidents developing an assistant that detects beforehand dangerous situations and extends in the time and in the space the help to drivers in an environment. The assistant is a cooperative system based on $\mathrm{V} 2 \mathrm{~V}$ and $\mathrm{I} 2 \mathrm{~V}$ communications.

In Asia, the Japan Automobile Research Institute (JARI) includes a research group in intelligent transportation systems. The efforts of this institute are focused on dis-

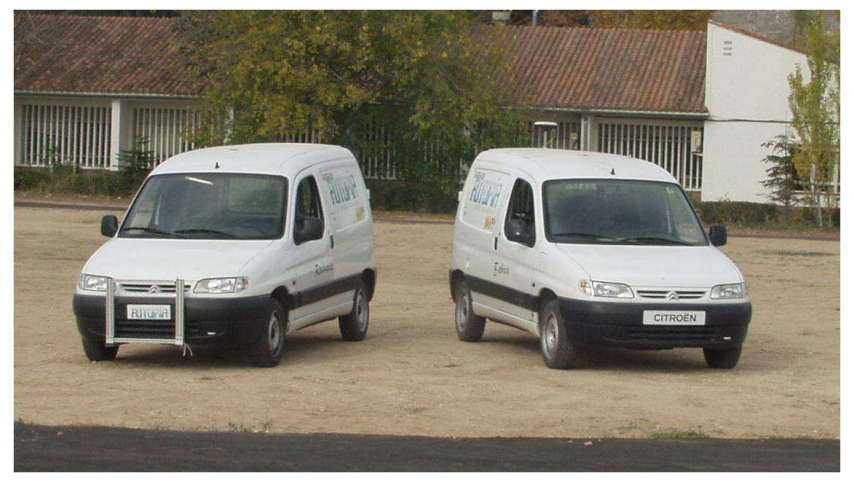

Fig. 2. Electric Citroen Berlingo vans.

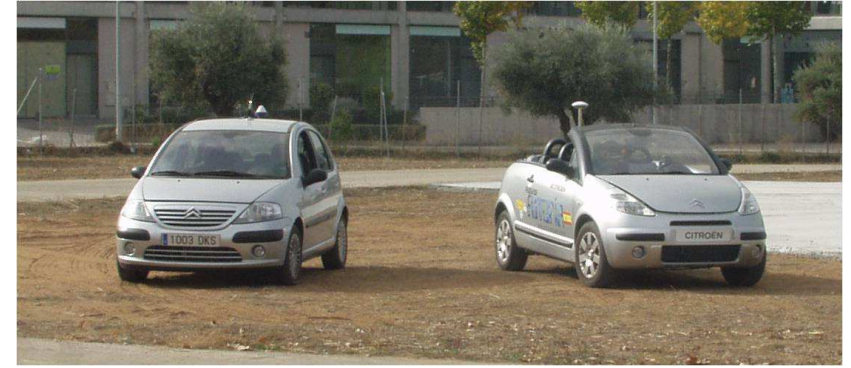

Fig. 3. Convertible Citroen C3 Pluriel fully automated and Citroen C3.

cussing the future perspective for the new motorized transportation society inside the new technological era. At the same time, they propose specific systems such as probe information systems in order to reach the new motorized society, conducing research and development in cooperation with engineers in various fields. The experiments are validated and verified by studying the social acceptance. Another important program participating in intelligent vehicle research is Japan's Advanced Cruise-Assist Highway Systems Research Association (AHSRA). Its goal is to develop the technology needed in order to support cooperative vehicle-highway safety systems. These systems aim to link Automated Highway Systems (AHS) research with Advanced Safety Vehicle (ASV) research through the use of $\mathrm{I} 2 \mathrm{~V}$ and $\mathrm{V} 2 \mathrm{~V}$ communications.

We here described an architecture to allow the coexistence among autonomous and manual driving vehicles based on a V2I architecture. This approach is based on low cost wireless communication systems. A central station coordinates the information interchange among vehicles within its influence area and, in function of their trajectory, decides to transfer the control to the next central station. In this way, a continuous data traffic flow can be possible among vehicles and cooperative maneuvers can be extended in the space.

The article is structured as follows. We introduce a review of the AUTOPIA group works in Sec. 2. The proposed architecture is defined in Sec. 3. Sec. 4. presents the physical implementation of the proposed architecture. An experiment to show the feasibility of the architecture is described in Sec. 5 and some concluding remarks and future works are given in Sec. 6 .

\section{REVIEW OF THE GROUP WORK}

The objective of AUTOPIA group consists on the development of an open architecture to carry out an automatic driving system able to manage a set of car maneuvers in the same way human drivers do. For that purpose, it deals with sensorial information and wireless communication as main sensorial inputs and manages the three fundamental actuators in a vehicle: throttle, brake and steering wheel.

First, two electric Citroen Berlingo vans were fully automated from 1998 to 2002 (García and de Pedro, 2000; Naranjo et al., 2003) (Fig. 2). These vans were equipped with a power stage -developed by the IAI-CSIC- able to control a DC motor attached to the steering wheel bar. Another DC motor was coupled to the brake pedal in order to act over it. Finally, a card was developed to read a pulse 


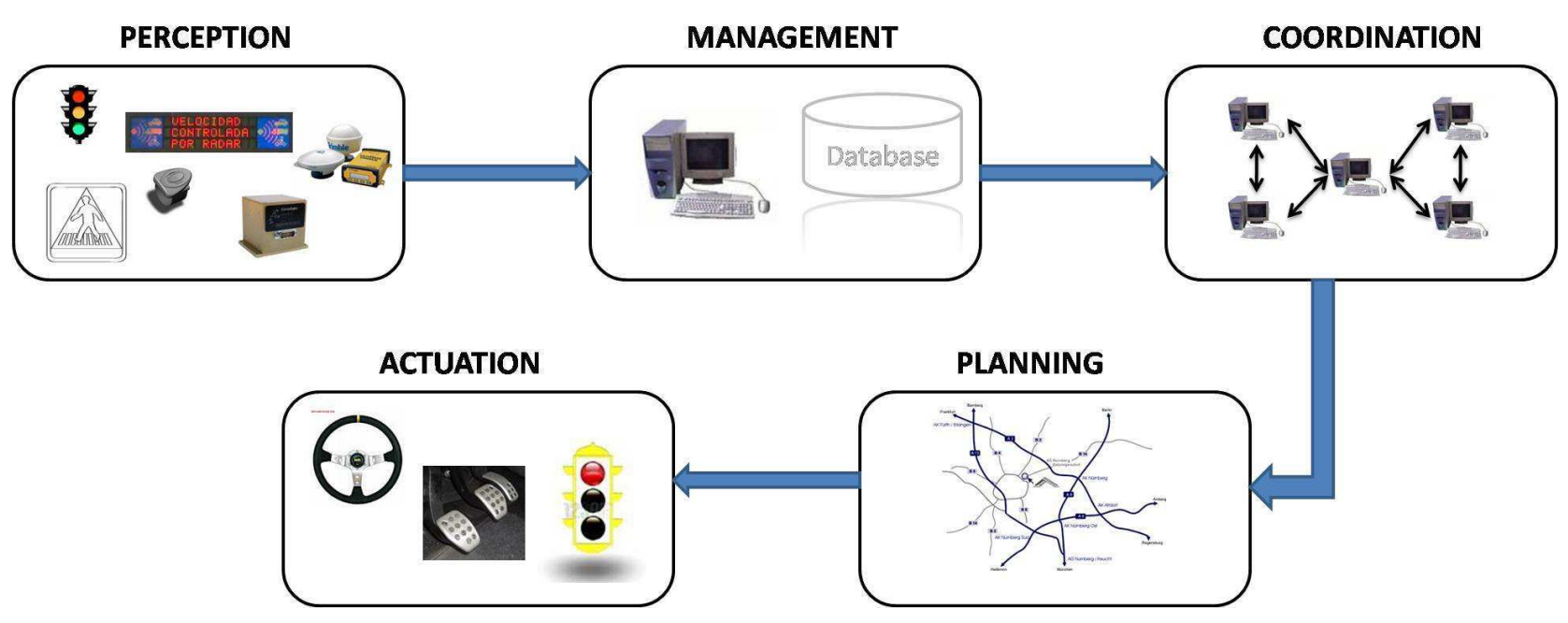

Fig. 4. V2I proposed architecture.

train coming from the wheels and so acquire the vehicle's speed. As output, it sent an analogue signal to control the throttle pedal. This hardware implementation has been replaced in recent years with new control devices (Perez et al., 2009). Presently, a power controller stage connected to the main PC via Ethernet is used in order to carry out the control of the steering wheel as well as that of the brake pedal. A CAN bus I/O device is used to read the signal coming from the wheel sensors and send the control signal to the throttle.

From 2005, a new vehicle has joined to the AUTOPIA group's fleet: a convertible Citroen C3 Pluriel (Fig. 3). This vehicle has been fully automated too. The main difference with respect to the vans is in the brake control implementation where a electro-hydraulic braking system was developed (Milanés et al., 2010a). At the end of 2006, another Citroen C3 was joined. It is equipped with a DGPS and a wireless system that allow it to send its position and speed to the autonomous vehicle in order to perform cooperative maneuvers while being manually driven (Perez et al., 2009).

With these vehicles and a real-time-kinematic differential global positioning system (RTK-DGPS) and an inertial measurement unit (IMU) as main inputs (Milanés et al., 2008), different cooperative maneuvers based on V2V communication were performed. Among them, adaptive cruise control + Stop\&Go maneuvers (Naranjo et al., 2006), overtaking (Naranjo et al., 2008) or intersection management (Milanés et al., 2010b) were carried out. All the controllers were designed using fuzzy logic that is a soft-computing technique that allows to perform a good control of non-linear systems as a vehicle is.

The fuzzy controllers were only tested in case of two vehicles driving along the road and a point-to-point communication was enough to send the control parameters between cars. However, when more vehicles are included in the experiments, a point-to-point communication can substantially increase the data traffic flow. This paper tackles this problem and a V2I architecture is presented to improve the obtained results.

\section{PROPOSED ARCHITECTURE}

Point-to-point communications cause an exponential number of opened communication channels in case of a lot car are driving in the same area. We here present a wide area architecture based on five levels to reduce the number of communication in order to perform a safety and efficient system.

In order to develop a wide area control system to improve the local area control used up to now, we must bear in mind:

1. A central unit of control must exist inside every local area that takes charge managing everything what happens inside it.

2. Every vehicle must know the information of the vehicles that they find in the same local area.

3. Existence of a common zone among local stations to assure the commutation of one zone to another one without loss of information.

4. Communication of the unit of local control with the local surrounding units in order to exchange the information of the vehicles that they find in the common zones.

Bearing this in mind, the proposed architecture (Fig. 4) is divided in five steps:

(1) Perception in this stage, all the sensorial information is sent from the vehicles - car, trucks, motorbikes, ...- and the infrastructure -traffic signals, light panels,...- to the local control station (LCS). From the vehicle's standpoint these information can come from Global Navigation Satellite Systems (GNSS), Inertial Measurement Unit (IMU), compass or any sensor that can be used (i.e. CAN bus) in the vehicles. From the infrastructure's standpoint one can use sensors placed at it -i.e. Zigbee or RFID systems- to obtain extrainformation. The LCS is limited by the area that can be covered by the local communication system.

(2) Management all the sensorial information received by the LCS is analyzed and efficiently sorted, that is, taking into account the traffic state and the number 


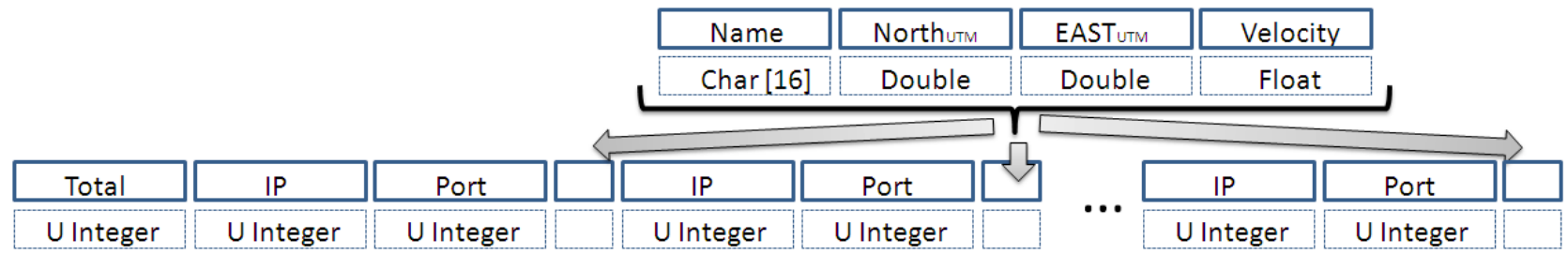

Fig. 5. Communication frame from vehicle to infrastructure and vice versa.

of vehicles driving in the area, find the best way to solve the risk traffic situation.

(3) Coordination in this stage, all the LCS send to the adjacent LCSs the information of the vehicle that are driving in common areas. Taking into account this information, the LCS will send to the vehicles and the infrastructure the data information in order to permit safety maneuvers.

(4) Planning with the information coming from the LCSs, the vehicles and the infrastructure evaluate the conditions and choose the best alternative in order to improve the traffic flow.

(5) Actuation in the last stage, the options selected in the previous stage are sent to the actuators. In case of a traffic light can be to change the light or in case or a vehicle can be to modify the speed.

\section{PHYSICAL IMPLEMENTATION}

From the functional point of view, the main problem of this architecture arise when a change of LCS is done. The solution adopted is based on a commutation structure previously defined.

Two different messages are generated. One of them is to send data from the vehicles to the LCS. (upper part of Fig. 5). This message contains the Name of the vehicle that is defined as a char, the position of the vehicle in Universal Transverse Mercator (UTM) North and East coordinates as doubles and the speed of the vehicle in kilometers per hour as float. The other one is the message send from the LCS to the vehicles and infrastructure. This message includes a header named 'Total' as unsigned integer that indicates the number of vehicles or devices that are included in the frame. Next, the IP is sent in order to know which were already connected, which are new or which are out of the interest area. Next, the

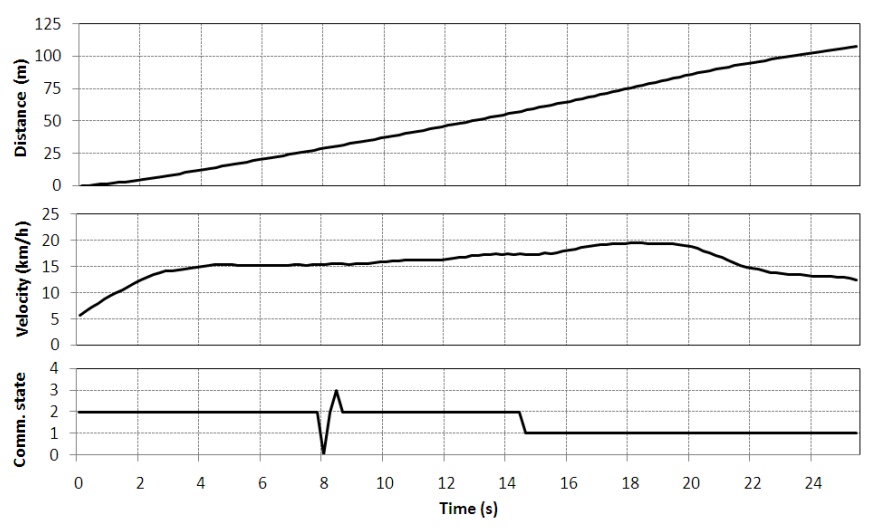

Fig. 6. Experiment to check the LCS change behavior. communication Port is sent as an unsigned integer. Finally, the frame with the actual characteristics of the vehicle or devices are sent. There are the same number of frames as number of vehicles/device are in the interest area.

From the programming point of view, we consider a bidirectional communication between the vehicle and the LCS. A client socket is installed in the vehicles and a server socket in the LCS. When a vehicle is close to leave the control area of an LCS and enters in the common area, a new message is included at the end of the frame with the IP of the new LCS. While the common area, the vehicle changes from an LCS to the other one and can continue without problems.

The tests carried out in order to check the behavior of the commutation system suggest that no delays are introduced when a change between two consecutive LCSs is done. From the safety point of view, the risk about a possible outage in the LCS change is similar than a failure in the WiFi connection.

Fig. 6 shows a test where only one vehicle is driven along the circuit by a human driver. The goal is to check the speed in the change of LCS. The upper part of the figure shows the distance covered by the car during the test. In the middle part the speed of the vehicle is plotted. The lower part presents the communication state from the vehicle point of view where: '0' indicates that the vehicle is disconnected of any LCS, '1' represents that the vehicle is connected to the LCS that cover the upper part of the circuit, '2' indicates the vehicle is receiving the signal from the LCS that cover the lower part and ' 3 ' represents that a delay in the communications has occurred.

The change between the LCSs is carried out around second 15 and no data are lost. One can appreciate how a WiFi signal failure occur around second 8 and. Two control cycles later, a delay in the communication with some data loss occurs when the signal is recovered. More trials were conducted and the duration either signal loss or delay has always been lower than three control cycle. Since the control cycle is triggered every 200 milliseconds, a possible loss in the signal lower than one second is assumed. Should longer failures occur, the system should be stopped through an emergency system.

\section{RELATED EXPERIMENT}

We here present the experiment that was conducted in order to test in a real environment the proposed architecture. First, the private driving circuit at the IAI-CSIC facilities is presented. Second, the experiment that was performed in order to demonstrate the behavior of the system is explained. 


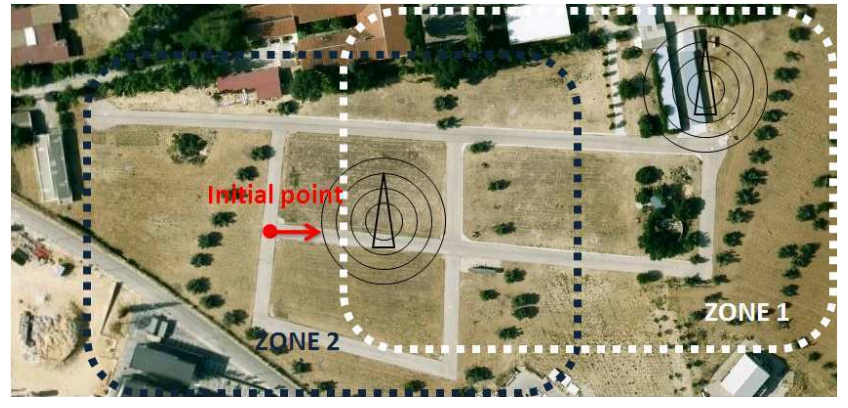

Fig. 7. Experimental area at the IAI-CSIC facilities.

The trial was carried out with two of the vehicles of the AUTOPIA group: the convertible C3 Pluriel and the C3. The former is fully automated and the latter is equipped with a DGPS and a wireless card in order to send the position and the speed to the LCS.

\subsection{Experimentation area}

The AUTOPIA group has a private driving circuit. It consists on a set of roads that emulate urban conditions. It includes roundabouts, crossroads, bends and straight stretches. This area has a longitude over one kilometer. Since the vehicles are equipped with a WiFi communication system, is impossible to cover all the circuit with only one LCS. For instance, two different LCSs were installed in the circuit (Fig. 7). Taking into account the characteristics of the experimental area, one of them was located in the terraced flat of one of the buildings of the IAI-CSIC and the other one was located in a new central station placed near the crossroad.

Each one of the LCS covers a different area but, we previously explained in section III, a common area must be defined in order to permit the change between two consecutive LCSs. In Fig. 7, one can appreciate the definition of each one of this area and the common one.

\subsection{Test}

To demonstrate the behavior of the architecture presented in this paper, a real experiment was performed with two vehicles. A follow the leader vehicle is carried out. Taking into account the two zones previously defined, the goal is to perform the LCS change transparently to the vehicles. The initial point of the vehicles's trajectory is marked in Fig. 7. Since the goal is to demonstrate the behavior of the change between two consecutive LCSs, we use only that street in order to test it. The length of the street is around 200 meters. The vehicles begin the maneuver at the end of the street connected to the LCS that cover the Zone 2 and, when we enter in the common area, the commutation from the Zone 2 to the Zone 1 is done.

During the experiment, the leading car was driven by a human driver. The longitudinal control -brake and throttle pedals- in the trailing vehicle was done autonomously whereas the lateral control -steering wheel- was done by a human driver. The management of the brake pedal as well as the throttle pedal were controlled via an on-board PC generated signals with a fuzzy controller in the high level control.
The results obtained from the experiment are shown in Fig. 8. The top graph shows the distance covered by the autonomous vehicle. The next to top graph shows the speed of the leading (grey) and of the trailing (black) cars, and the next to bottom graph shows the gap in meters between the cars. The bottom graph shows the value of the communication state with the same meaning for each one of the possible values.

One can appreciate how different delays are registered while the vehicle is driven across the Zone 2 in four times. During LCS's commutation from Zone 2 to Zone 1 a loss of signal during two control cycle occurs. As we said before, the gap defined between vehicle can assume it and, when the connection to the LCS located in the Zone 1 is done, the control system continues working well. From that instant to the end, no more failures in the communication system are detected.

\section{CONCLUSIONS AND FUTURE WORKS}

In this paper, a V2I-based architecture for communication among vehicles is presented. Taking into account the advance in autonomous vehicle, we consider that, at any time, a coexistence among autonomous vehicle and vehicle driven by human drivers occur. This paper deals with an architecture able to support any kind of unexpected circumstance. With this purpose, an LCS has been designed as it also has been a communication system to exchange information among all the adjacent LCSs in order to have available all the crucial data that permits an increase in the safety. The design is done to permit, with a minimum modification, the inclusion of a new vehicle or infrastructure device in the architecture in an easy way.

The implementation presented in this paper was done in a real scenario and with real vehicle to check the behavior of the system. Two vehicles were used to study the information exchange between them. A follow the leader vehicle was presented with the inclusion of an LCS change. Since we are able to carry out this trial, one

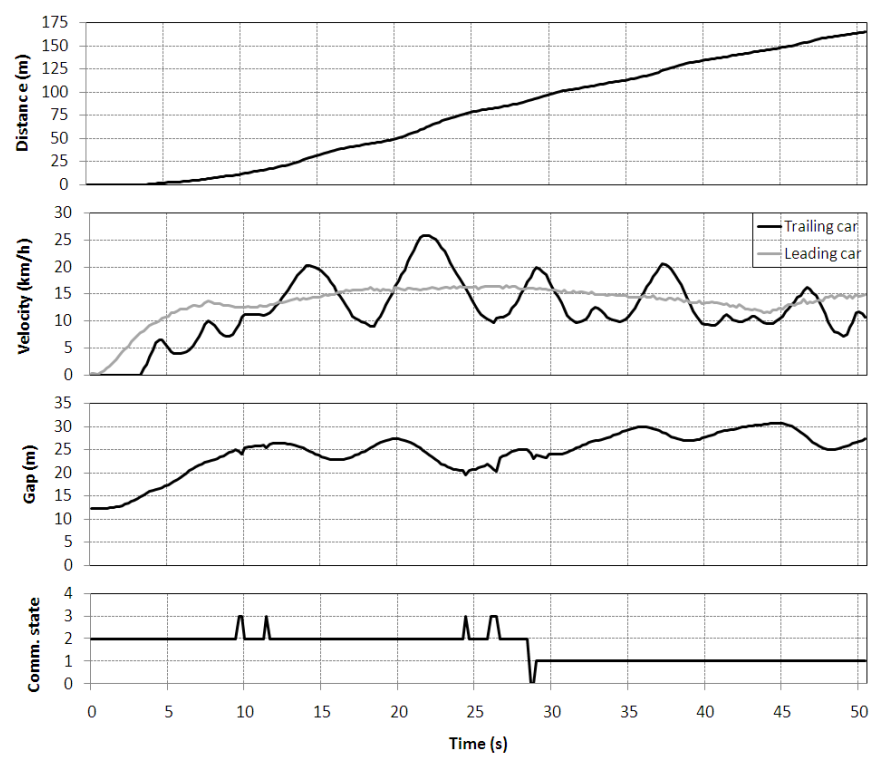

Fig. 8. Experimental results. 
can oversize this result and think that it will be possible to commute among an indefinite numbers of LCSs with enough safety. The test was done with WiFi system but it would be as easy to test with any kind of communication system as WiMax, GPRS, etc.

The next goal is to introduce more vehicles in the test area and check the limit of the LCS in function of the number of signal received and the signal that are sent to the vehicles and the infrastructure device. Indeed, we expect to carry out trials with different communication systems to check which is the best option to implement the presented architecture.

\section{ACKNOWLEDGEMENTS}

The authors are grateful to the CYCIT (Spain), Plan Nacional (Spain), and MICINN (Spain) for support under the GUIADE (P9/08), TRANSITO (TRA2008-06602C03-01), and City-Elec (PS-370000-2009-4) projects, respectively, for the development of this work.

\section{REFERENCES}

Biswas, S., Tatchikou, R., and Dion, F. (2006). Vehicle-tovehicle wireless communication protocols for enhancing highway traffic safety. IEEE Communications Magazine, 44(1), 74-82.

Chen, W. and Cai, S. (2005). Ad hoc peer-to-peer network architecture for vehicle safety communications. IEEE Communications Magazine, 43(4), 100-107.

García, R. and de Pedro, T. (2000). First application of the orbex coprocessor: Control of unmanned vehicles. EUSFLAT-ESTYLF Joint Conference. Mathware and Soft Computing, 7(2-3), 265-273.

Milanés, V., González, C., Naranjo, J.E., Onieva, E., and de Pedro, T. (2010a). Electro-hydraulic braking system for autonomous vehicles. International Journal of Automotive Technology, 1(11), 89-95.

Milanés, V., Naranjo, J.E., Gonzalez, C., Alonso, J., and de Pedro, T. (2008). Autonomous vehicle based in cooperative gps and inertial systems. Robotica, 26(5), $627-633$.

Milanés, V., Pérez, J., Onieva, E., and González, C. (2010b). Controller for urban intersections based on wireless communications and fuzzy logic. IEEE Transactions on Intelligent Transportation Systems, 11(1), 243-248.

Naranjo, J.E., Gonzalez, C., Garcia, R., and de Pedro, T. (2006). Acc+stop\&go maneuvers with throttle and brake fuzzy control. IEEE Transactions on Intelligent Transportation Systems, 7(2), 213-225.

Naranjo, J.E., Gonzalez, C., Garcia, R., and de Pedro, T. (2008). Lane-change fuzzy control in autonomous vehicles for the overtaking maneuver. IEEE Transactions on Intelligent Transportation Systems, 9(3), 438-450.

Naranjo, J.E., Gonzalez, C., Reviejo, J., Garcia, R., and de Pedro, T. (2003). Adaptive fuzzy control for intervehicle gap keeping. IEEE Transactions on Intelligent Transportation Systems, 4(3), 132-142.

Perez, J., Gonzalez, C., Milanes, V., Onieva, E., Godoy, J., and de Pedro, T. (2009). Modularity, adaptability and evolution in the autopia architecture for control of autonomous vehicles. In Proc. IEEE International Conference on Mechatronics ICM 2009, 1-5.
Piao, J. and McDonald, M. (2008). Safety impacts of variable speed limits - a simulation study. In Proc. 11th International IEEE Conference on Intelligent Transportation Systems ITSC 2008, 833-837.

Singh, V., Singh, T., Langan, D., and Kumar, P. (2004). A framework for internet gis based computerized visitor information system for theme parks. In Proc. 7th International IEEE Conference on Intelligent Transportation Systems, 679-683.

Vahidi, A. and Eskandarian, A. (2003). Research advances in intelligent collision avoidance and adaptive cruise control. IEEE Transactions on Intelligent Transportation Systems, 4(3), 143-153.

Vivo, G., Dalmasso, P., and Vernacchia, F. (2007). The european integrated project "safespot"-how adas applications co-operate for the driving safety. In Proc. IEEE Intelligent Transportation Systems Conference ITSC 2007, 624-629.

Wu, S.J., Chiang, H.H., Perng, J.W., Chen, C.J., Wu, B.F., and Lee, T.T. (2008). The heterogeneous systems integration design and implementation for lane keeping on a vehicle. IEEE Transactions on Intelligent Transportation Systems, 9(2), 246-263. 